\title{
A CLASS OF MULTIVALENT FUNCTIONS
}

\author{
BY \\ A. W. GOODMAN AND M. S. ROBERTSON
}

1. Introduction. It has long been conjectured that if

$$
f(z)=b_{1} z+b_{2} z^{2}+\cdots+b_{n} z^{n}+\cdots
$$

is regular and univalent for $|z|<1$, then

$$
\left|b_{n}\right| \leqq n\left|b_{1}\right|
$$$$
n=2,3, \cdots .
$$

This inequality has been proved for $n=2$ and $n=3$, and for special subclasses of the family of univalent functions (1.2) has been proved for all $n$. In particular, if all the coefficients in (1.1) are real then (1.2) holds and is sharp for all positive integers $n$. In fact Rogosinski [8](1), Dieudonné [2] and Szász [9] have proved (1.2) when all the coefficients are real and $f(z)$ satisfies a weaker condition than univalency, namely that for $|z|<1, \Im f(z)>0$ when and only when $\Im z>0$. Such a function is called typically-real in the unit circle.

The situation is quite different for the class $V(p)$ of functions (1.1) which are regular and multivalent of order $p$ ( $p$-valent) in $|z|<1$. Almost without exception, the previous investigations were concerned only with the order of magnitude of the coefficients $b_{n}$, and the most recent result due to Biernacki [1] that

$$
\left|b_{n}\right| \leqq A(p) \max \left\{\left|b_{1}\right|,\left|b_{2}\right|, \cdots,\left|b_{q}\right|\right\} n^{2 p-1}, \quad q \text { zeros in }|z|<1,
$$

could only be accepted as a first and rough approximation of the facts.

Quite recently A. W. Goodman [3] conjectured that if $f(z) \in V(p)$ then for $n>p$

$$
\left|b_{n}\right| \leqq \sum_{k=1}^{p} \frac{2 k(n+p) !}{(p+k) !(p-k) !(n-p-1) !\left(n^{2}-k^{2}\right)}\left|b_{k}\right| .
$$

This expression becomes (1.2) when $p=1$, and when $p=2$ and $n=3$ (1.3) becomes

$$
\left|b_{3}\right| \leqq 5\left|b_{1}\right|+4\left|b_{2}\right|
$$

an inequality which has been proved valid if $f(z) \in V(2)$, has all coefficients real, and in addition is starlike with respect to the origin [4].

We shall prove, in the following work, that for a certain class of functions $T(p)$, to be defined precisely in $\S 2$, the inequality (1.3) is valid and further (1.3)

Presented to the International Congress of Mathematicians, September 2, 1950; received by the editors April 21, 1950.

(1) Numbers in brackets refer to the bibliography at the end of this work. 
is sharp in all of the $p$ variables, namely for every set $\left\{\left|b_{1}\right|,\left|b_{2}\right|, \cdots,\left|b_{p}\right|\right\}$ not all zero there is an $f(z) \in T(p)$ such that (1.3) holds with the equality sign.

The class of functions $T(p)$ was first introduced by M. S. Robertson [5] [6] and is essentially the class of functions (1.1) with the real coefficients such that $\Im f(z)$ changes sign $2 p$ times on $|z|=r$ for some range $0<\rho<r<1$. This class of functions is a natural generalization of Rogosinski's typically-real functions and we shall call functions of this class typically-real functions of order $p$. We observe that $T(p)$ contains functions of arbitrarily high valence for any fixed $p$, and contains a wide class of $p$-valent functions, in particular $T(p)$ contains all starlike $p$-valent functions with real coefficients.

For $f(z) \in T(p)$ Robertson [6] has proved that

$$
\left|b_{n}\right|<A(p) \max \left\{\left|b_{1}\right|,\left|b_{2}\right|, \cdots,\left|b_{p}\right|\right\} n^{2 p-1}, \quad n>p \geqq 1,
$$

and that $f(z)$ can be represented in the form $\lim g(z)$, where

$$
g(z)=\frac{z^{p} F(z)}{1-z^{2}} \prod_{j=2}^{p}\left(1-2 z \cos \theta_{j}+z^{2}\right)^{-1}
$$

and $F(z)$ is regular for $0<|z| \leqq 1$, has a pole of order $p-1$ at $z=0, \Re F\left(e^{i \theta}\right)$ $\geqq 0$, and $\theta_{j}$ are certain real parameters which depend on $f(z)$.

In attempting to prove (1.3) the authors were able to obtain (1.3) for the cases $p=2$ and $p=3$ directly from (1.6) making use of a lemma of Robertson $[6$, p. 514]. However the calculations are quite long and for $p>3$ they become very difficult to handle due to the appearance of trigonometric functions of $p-1$ variables. This difficulty is avoided by using induction on $p$, and this is the method of proof presented here.

2. Preliminary considerations. We first give a few definitions and state without proof a number of obvious lemmas which will be useful.

Definition 1. The harmonic function $v(r, \theta)$ is said to have a change of sign at $\theta=\theta_{j}$ if there exists an $\epsilon>0$ such that for $0<\delta<\epsilon$

$$
v\left(r, \theta_{j}+\delta\right) v\left(r, \theta_{j}-\delta\right)<0 .
$$

Note that in (2.1) $r$ is constant, and $\theta_{j}$, which is fixed for a given $v(r, \theta)$ and a given $r$, will in general depend on $r$.

Definition 2. $\Im f(z)=v(r, \theta)$ is said to change sign $q$ times on $|z|=r$ if there are $q$ values of $\theta, \theta_{1}, \theta_{2}, \cdots, \theta_{q}$ such that

(a) Inequality (2.1) holds for each $\theta_{j}, j=1,2, \cdots, q$,

(b) $\theta_{j} \not \equiv \theta_{k}(\bmod 2 \pi)$ if $j \neq k$,

(c) if $\theta_{s}$ is any value of $\theta$ for which $v(r, \theta)$ has a change of sign then for one of the $\theta_{j}, j=1, \cdots, q$,

$$
\theta_{s} \equiv \theta_{j}(\bmod 2 \pi)
$$

Lemma 1. If $v_{1}(r, \theta)$ and $v_{2}(r, \theta)$ both have a change of sign at $\theta_{j}$, then the product $v_{1}(r, \theta) v_{2}(r, \theta)$ does not have a change of sign at $\theta_{j}$. If $v_{1}(r, \theta)$ has a change 
of sign at $\theta_{j}$ and if $v_{2}(r, \theta)$ does not have a change of sign at $\theta_{j}$ then the product has a change of sign at $\theta_{j}$.

Lemma 2. Let $f(z)$ be regular for $|z| \leqq 1$. Then for each $r, 0<r \leqq 1, \Im f(z)$ changes sign an even number of times.

Definition 3. The function $f(z)$

$$
f(z)=\sum_{n=1}^{\infty} c_{n}^{(p)} z^{n}
$$

is said to belong to the class $T_{1}(p), p$ a positive integer, if real,

(a) $f(z)$ is regular for $|z| \leqq 1$, and all the coefficients $c_{n}^{(p)}, n=1,2, \cdots$ are

(b) $\Im f(z)$ has $2 p$ changes of sign on $|z|=1$.

Note that we have changed the notation slightly in going from equation (1.1) to (2.2). This is done to facilitate the proof by induction on $p$.

DefinItIon 4. $f(z)$ is said to belong to the class $T_{2}(p), p$ a positive in teger, if

(a) $f(z)$ is regular for $|z|<1$, and all the coefficients in (2.2) are real,

(b) there exists a $\rho<1$ such that for each $r$ in $\rho<r<1, \Im f(z)$ changes sign $2 p$ times on $|z|=r$.

Definition 5. $T(p)=T_{1}(p) \cup T_{2}(p)$. We call functions of the class $T(p)$, typically-real of order $p$.

Lemma 3. If $f(z) \in T_{2}(p)$, then $f(r z) \in T_{1}(p)$ for each $r$ in $\rho<r<1$.

Thus we need to prove (1.3) only for $f(z) \in T_{1}(p)$ since by letting $r \rightarrow 1$ this will imply that (1.3) is also valid for $f(z) \in T_{2}(p)$.

Lemma 4. If $f(z) \in T_{1}(p)$, then $\Im f\left(e^{i \theta}\right)$ changes sign at $\theta=0$ and $\theta=\pi$. If $\Im f\left(e^{i \theta}\right)$ changes sign at $\theta_{j}$, then it also changes sign at $-\theta_{j}$.

Lemma 5. If $\Im f(z)$ changes sign at $\theta=\theta_{j}$ and $A$ is any real constant, then $\Im(f(z)-A)$ changes sign at $\theta_{j}$.

Observe that if $f(z) \in T_{1}(p)$, then $f(z)-A \notin T(p)$ since we have required $f(0)=0$ for functions of this class.

THEOREM 1. If $f(z) \in T_{1}(p), p \geqq 2$, then there exists a real number $\nu$ such that if $g(z)$ is defined by

$$
g(z)=\frac{1-2 z \cos \nu+z^{2}}{z} f(z)-c_{1}^{(p)}=\sum_{n=1}^{\infty} c_{n}^{(p-1)} z^{n}
$$

then $g(z) \in T_{1}(p-1)$.

Proof. Let $k_{\nu}(z)=\left(1-2 z \cos \nu+z^{2}\right) / z$, then $k_{\nu}\left(e^{i \theta}\right)=2(\cos \theta-\cos \nu)$. Since $p \geqq 2, \Im f(z)$ changes sign at $\theta_{j}$ where $\theta_{j} \neq 0, \pi(\bmod 2 \pi)$. Let $\nu=\theta_{j}$. Then 
$2(\cos \theta-\cos \nu)$ changes sign exactly twice in $-\pi \leqq \theta \leqq \pi$, namely at $\theta_{j}$ and $-\theta_{j}$. Therefore by Lemma $1 \Im g\left(e^{i \theta}\right)=2(\cos \theta-\cos \nu) \Im f\left(e^{i \theta}\right)$ will not change sign at $\theta_{j}$ and $-\theta_{j}$, but changes sign at the other $2 p-2$ points where $\Im f\left(e^{i \theta}\right)$ changes sign and only at these points. Further $g(z)$ is regular for $|z| \leqq 1$ and $g(0)=0$. Therefore $g(z) \in T_{1}(p-1)$.

We remark that for $\nu \neq \theta_{j}, j=1,2, \cdots, 2 p, g(z)$ belongs to $T_{1}(p+1)$ instead of $T_{1}(p-1)$.

\section{The main theorem.}

THEOREM 2. If $f(z)$ of the form (2.2) is a member of the class $T(p)$ then for $n>p$

$$
\left|c_{n}^{(p)}\right| \leqq \sum_{k=1}^{p} D(p, k, n)\left|c_{k}^{(p)}\right|
$$

where

$$
D(p, k, n) \equiv \frac{2 k(n+p) !}{(p+k) !(p-k) !(n-p-1) !\left(n^{2}-k^{2}\right)},
$$

and this inequality is sharp in all the variables $\left|c_{1}^{(p)}\right|,\left|c_{2}^{(p)}\right|, \cdots,\left|c_{p}^{(p)}\right|$, that is, for each set $\left|c_{k}^{(p)}\right|, k=1,2, \cdots, p$, not all zero, there is an $f(z) \in T(p)$ for which the equality sign holds in (3.1).

Proof. As a consequence of Lemma 3, it will be sufficient to prove (3.1) for $f(z) \in T_{1}(p)$. Now the theorem is already known to be true for $p=1,[2],[8],[9]$, and so, using induction, we may proceed to assume (3.1) valid for index $p-1$, and show that, for each $p \geqq 2$, this implies that (3.1) is also valid for index $p$.

Let $f(z) \in T_{1}(p)$ be given by (2.2). By Theorem 1 we may select a real $\nu$ so that $g(z)$ given by

$$
g(z)=\frac{1-2 z \cos \nu+z^{2}}{z} f(z)-c_{1}^{(p)}=\sum_{n=1}^{\infty} c_{n}^{(p-1)} z^{n}
$$

belongs to the class $T_{1}(p-1)$. By equating coefficients we have

$$
c_{k}^{(p-1)}=c_{k+1}^{(p)}-2 c_{k}^{(p)} \cos \nu+c_{k-1}^{(p)}, \quad k=1,2, \cdots,
$$

where for convenience we have set $c_{0}^{(p)}=0$. Clearly

$$
\left|c_{k}^{(p-1)}\right| \leqq\left|c_{k+1}^{(p)}\right|+2\left|c_{k}^{(p)}\right|+\left|c_{k-1}^{(p)}\right|, \quad k=1,2, \cdots .
$$

Solving (3.3) for $f(z)$ we find that

$$
\frac{z\left(g(z)+c_{1}^{(p)}\right)}{1-2 z \cos \nu+z^{2}}=f(z)=\sum_{n=1}^{\infty} c_{n}^{(p)} z^{n}
$$

and hence on equating coefficients 


$$
c_{n}^{(p)}=\sum_{k=0}^{n-1} c_{k}^{(p-1)} \frac{\sin (n-k) \nu}{\sin \nu}, \quad n=1,2, \cdots,
$$

where, although $g(0)=0$, we have adopted the convention that $c_{0}^{(p-1)}=c_{1}^{(p)}$ in order to simplify the expression (3.7). For $n>p$ we may write

$$
c_{n}^{(p)}=S_{1}+S_{2}
$$

where

$$
S_{1}=\sum_{k=0}^{p-1} c_{k}^{(p-1)} \frac{\sin (n-k) \nu}{\sin \nu}
$$

and

$$
S_{2}=\sum_{k=p}^{n-1} c_{k}^{(p-1)} \frac{\sin (n-k) \nu}{\sin \nu} .
$$

Substituting (3.4) in (3.9) we find

$$
\begin{aligned}
S_{1} & =c_{1}^{(p)}\left\{\frac{\sin n \nu}{\sin \nu}-2 \cos \nu \frac{\sin (n-1) \nu}{\sin \nu}+\frac{\sin (n-2) \nu}{\sin \nu}\right\} \\
& +c_{2}^{(p)}\left\{\frac{\sin (n-1) \nu}{\sin \nu}-2 \cos \nu \frac{\sin (n-2) \nu}{\sin \nu}+\frac{\sin (n-3) \nu}{\sin \nu}\right\}+\cdots \\
& +c_{p-2}^{(p)}\left\{\frac{\sin (n-p+3) \nu}{\sin \nu}-2 \cos \nu \frac{\sin (n-p+2) \nu}{\sin \nu}+\frac{\sin (n-p+1) \nu}{\sin \nu}\right\} \\
& +c_{p-1}^{(p)}\left\{\frac{\sin (n-p+2) \nu}{\sin \nu}-2 \cos \nu \frac{\sin (n-p+1) \nu}{\sin \nu}\right\} \\
& +c_{p}^{(p)} \frac{\sin (n-p+1) \nu}{\sin \nu} .
\end{aligned}
$$

Since we have identically

$$
\sin (m+1) \nu-2 \cos \nu \sin m \nu+\sin (m-1) \nu=0,
$$

the expression for $S_{1}$ collapses to

$$
S_{1}=-c_{p-1}^{(p)} \frac{\sin (n-p) \nu}{\sin \nu}+c_{p}^{(p)} \frac{\sin (n-p+1) \nu}{\sin \nu},
$$

so that

$$
\left|S_{1}\right| \leqq(n-p)\left|c_{p-1}^{(p)}\right|+(n-p+1)\left|c_{p}^{(p)}\right| .
$$

We now assume that (3.1) is valid for index $p-1$, that is we assume that 
for $k=p, p+1, \cdots$

$$
\left|c_{k}^{(p-1)}\right| \leqq \sum_{s=1}^{p-1} D(p-1, s, k)\left|c_{s}^{(p-1)}\right| .
$$

From (3.10), (3.14), and (3.5) we have

$$
\begin{aligned}
& \left|S_{2}\right| \leqq \sum_{k=p}^{n-1}(n-k) \sum_{s=1}^{p-1} D(p-1, s, k)\left|c_{s}^{\left(p_{i}-1\right)}\right| \\
& \left|S_{2}\right| \leqq \sum_{k=p}^{n-1}(n-k) \sum_{s=1}^{p-1} D(p-1, s, k)\left(\left|c_{s+1}^{(p)}\right|+2\left|c_{s}^{(p)}\right|+\left|c_{s-1}^{(p)}\right|\right) .
\end{aligned}
$$

Finally (3.8), (3.13), and (3.15) give for $n=p+1, p+2, \cdots$

$$
\begin{aligned}
\left|c_{n}^{(p)}\right| \leqq & (n-p)\left|c_{p-1}^{(p)}\right|+(n-p+1)\left|c_{p}^{(p)}\right| \\
& +\sum_{k=p}^{n-1}(n-k) \sum_{s=1}^{p-1} D(p-1, s, k)\left(\left|c_{s+1}^{(p)}\right|+2\left|c_{s}^{(p)}\right|+\left|c_{s-1}^{(p)}\right|\right) .
\end{aligned}
$$

We now define $D^{*}(p, k, n)$ by the right side of the inequality (3.16), namely through the identity

$$
\begin{aligned}
& \sum_{k=1}^{p} D^{*}(p, k, n)\left|c_{k}^{(p)}\right| \equiv(n-p)\left|c_{p-1}^{(p)}\right|+(n-p+1)\left|c_{p}^{(p)}\right| \\
& +\sum_{k=p}^{n-1}(n-k) \sum_{s=1}^{p-1} D(p-1, s, k)\left(\left|c_{s+1}^{(p)}\right|+2\left|c_{s}^{(p)}\right|+\left|c_{s-1}^{(p)}\right|\right) .
\end{aligned}
$$

It is obvious that if we can prove

$$
D^{*}(p, k, n)=D(p, k, n), \quad 1 \leqq k \leqq p<n,
$$

the proof of (3.1) will be completed. It is not too difficult to prove (3.18) directly from the definition (3.17), for the cases $p=2$ and $p=3$, and with somewhat more effort (3.18) can be shown for arbitrary $p$ and $n$ in the case $k=p$. However in the completely general case $1 \leqq k \leqq p<n$ the manipulations become extremely complicated, and so we resort to a device.

4. Proof that $D^{*}(p, k, n)=D(p, k, n)$. Essentially what we shall do will be to show that if $\nu=0$ in the work of the preceding section then $f(z)$ is just one of a certain family of functions whose coefficients are given by (3.1) with the equality sign. But (3.16) will also hold simultaneously with the equality sign and hence (3.18). To bring out the structure of the proof more clearly, we have numbered the equations of this section in such a way that whenever the decimal part is the same as that for an equation in $\$ 3$, the latter equation is similar in form to the one obtained by setting $\nu=0$ in the former.

LemMA 6. Let $u=z /(1-z)^{2}$ and let $c_{1}, c_{2}, \cdots, c_{p}$ be any set of positive 
numbers. Then there is a unique function $F(z)$ such that

$$
F(z)=\sum_{n=1}^{\infty} b_{n}^{(p)} z^{n}=a_{1}^{(p)} u+a_{2}^{(p)} u^{2}+\cdots+a_{p}^{(p)} u^{p}
$$

where

$$
b_{k}^{(p)}=(-1)^{p-k} c_{k}
$$

$$
k=1,2, \cdots, p
$$

and for this function

$$
b_{n}^{(p)}=\sum_{k=1}^{p} D(p, k, n) c_{k}, \quad n=p+1, p+2, \cdots
$$

Conversely if $G(z)$ is known to be a pth degree polynomial in $u$, that is if

$$
G(z)=a_{1}^{(p)} u+a_{2}^{(p)} u^{2}+\cdots+a_{p}^{(p)} u^{p}=\sum_{n=1}^{\infty} b_{n}^{(p)} z^{n}
$$

and if $\operatorname{sgn} b_{k}^{(p)}=(-1)^{p-k}, k=1,2, \cdots, p$, then the $c_{k}$ defined by (4.02) are all positive and (4.03) holds.

Proof. Although not expressly stated, this lemma was proved in [3]. There it was shown that in (4.01) the $a_{k}^{(p)}$ are uniquely determined by the $b_{k}^{(p)}, k=1,2, \cdots, p$, indeed (equation (3.8) of [3])

$$
a_{m}^{(p)}=\sum_{k=1}^{m}(-1)^{k+m} b_{k}^{(p)} \frac{2 k(2 m-1) !}{(m+k) !(m-k) !}, \quad m=1,2, \cdots, p .
$$

Hence for $n>p, b_{n}^{(p)}$ is uniquely determined by $b_{k}^{(p)}, k=1,2, \cdots, p$, indeed (equations (3.9) and (3.11) of [3])

$$
b_{n}^{(p)}=\sum_{k=1}^{p}(-1)^{p-k} b_{k}^{(p)} D(p, k, n), \quad n=p+1, p+2, \cdots
$$

Combining this with (4.02) gives (4.03). Conversely in (4.04), the $b_{n}^{(p)}$ are obviously uniquely determined by the $a_{k}^{(p)}, k=1,2, \cdots, p$. Just as before (4.05) and (4.06) hold, and with $c_{k}$ determined from (4.02), (4.03) again follows.

We apply Lemma 6 to the proof of (3.18) thus. Select any set of positive numbers $c_{1}, c_{2}, \cdots, c_{p}$ and with this fixed set construct the unique $F(z)$ as indicated in the first part of the lemma. For this $F(z)$ we have (4.01), (4.02), and (4.03). With this $F(z)$, define $G(z)$ by

$$
G(z)=\frac{(1-z)^{2}}{z} F(z)-b_{1}^{(p)}=\sum_{n=1}^{\infty} b_{n}^{(p)} z^{n}
$$

From (4.3), (4.01), and $b_{1}^{(p)}=a_{1}^{(p)}$ it follows that 


$$
G(z)=a_{2}^{(p)} u+a_{3}^{(p)} u^{2}+\cdots+a_{p}^{(p)} u^{p-1} .
$$

By equating coefficients in (4.3) we have

$$
b_{k}^{(p-1)}=b_{k+1}^{(p)}-2 b_{k}^{(p)}+b_{k-1}^{(p)}, \quad k=1,2, \cdots,
$$

where for convenience we have set $b_{0}^{(p)}=0$. Further from (4.02) it is clear that

$$
\left|b_{k}^{(p-1)}\right|=c_{k+1}+2 c_{k}+c_{k-1}, \quad k=1,2, \cdots, p-1,
$$

and $\operatorname{sgn} b_{k}^{(p-1)}=(-1)^{p-1-k}, k=1,2, \cdots, p-1$. This together with (4.07) shows that $G(z)$ satisfies the conditions of the second half of Lemma 6 with index $p-1$, and so for the coefficients of $G(z)$ we have

$$
b_{k}^{(p-1)}=\sum_{s=1}^{p-1} D(p-1, s, k)\left|b_{s}^{(p-1)}\right|, \quad k=p, p+1, \cdots
$$

Solving (4.3) for $F(z)$ gives

$$
F(z)=\frac{z}{(1-z)^{2}}\left(G(z)+b_{1}^{(p)}\right)=\sum_{n=1}^{\infty} b_{n}^{(p)} z^{n},
$$

and hence on equating coefficients

$$
b_{n}^{(p)}=\sum_{k=0}^{n-1}(n-k) b_{k}^{(p-1)}, \quad n=1,2, \cdots,
$$

where for convenience we have set $b_{0}^{(p-1)}=b_{1}^{(p)}$. For $n>p$ we may write

$$
b_{n}^{(p)}=\sum_{k=0}^{p-1}(n-k) b_{k}^{(p-1)}+\sum_{k=p}^{n-1}(n-k) b_{k}^{(p-1)}=S_{1}^{\prime}+S_{2}^{\prime} .
$$

Using (4.4) in $S_{1}^{\prime}$ and recalling (4.02) we find

$$
\begin{aligned}
& S_{1}^{\prime}=-(n-p) b_{p-1}^{(p)}+(n-p+1) b_{p}^{(p)}, \\
& S_{1}^{\prime}=(n-p) c_{p-1}+(n-p+1) c_{p},
\end{aligned}
$$

and using (4.14) and (4.5) in $S_{2}^{\prime}$ we have

$$
S_{2}^{\prime}=\sum_{k=p}^{n-1}(n-k) \sum_{s=1}^{p-1} D(p-1, s, k)\left(c_{k+1}+2 c_{k}+c_{k-1}\right) .
$$

Finally (4.8), (4.13), and (4.15) give for $n>p$

$$
\begin{aligned}
b_{n}^{(p)}= & (n-p) c_{p-1}+(n-p+1) c_{p} \\
& +\sum_{k=p}^{n-1}(n-k) \sum_{s=1}^{p-1} D(p-1, s, k)\left(c_{k+1}+2 c_{k}+c_{k-1}\right) .
\end{aligned}
$$


But $b_{n}^{(p)}$ is also given by (4.03) for each $n>p$. Since the $c_{k}$ were arbitrary positive numbers, the coefficients of $c_{k}$ on the right side of (4.16) and (4.03) are identical. Hence $D^{*}(p, k, n)=D(p, k, n)$ and (3.1) is proved.

5. Proof that (3.1) is sharp. We first recall the following definition.

Definition 6. A function $f(z)$ is said to be $p$-valently starlike with respect to a point $\omega_{0}$, for $|z|<1$, if (a) $f(z)$ is regular and $p$-valent for $|z|<1$ and (b) if there exists a $\rho$ such that for each $r$ in $\rho<r<1$, the radius vector joining the point $\omega_{0}$ to $f\left(r e^{i \theta}\right)$ turns continuously in the counter-clockwise direction and makes $p$ complete revolutions as $\theta$ varies from 0 to $2 \pi$.

These starlike functions have been studied previously by Robertson and others. A rather complete list of references may be found in [4, footnote 16]. We have obviously the following lemma.

LemMa 7. If $f(z)$ is p-valently starlike with respect to a point on the real axis for $|z|<1$, and if all the coefficients are real then $f(z) \in T(p)$.

Let us now examine more closely the extremal functions constructed in [3]. To be specific we shall say that $F(z)$ is an extremal function if there is a set of nonnegative constants $c_{k}, k=1,2, \cdots, p$, not all zero, such that $F(z)$ is given by (4.01) and (4.02). It was proved in [3] that for these extremal functions (3.1) holds with the equality sign. It remains to show only that $F(z) \in T(p)$.

It was further proved in $[4$, Theorem 12] that these extremal functions are all starlike with respect to the origin, but it was not proved in [4] that $F(z)$ is starlike of order $p$, although this result appears to be intuitively evident and was announced there. To remedy this defect we remark that (4.02) and (4.05) together show that $\operatorname{sgn} a_{m}^{(p)}=(-1)^{p+m}$. Hence from (4.01), $F(z) \neq 0$ for $u<0$. But $F(z)$ as a polynomial of pth degree in $u$ has exactly $p$ zeros $u_{k}$ and since each zero $u_{k}$ is not on the line $-\infty<u<-1 / 4$, it is the image of exactly one $z_{k}$ in $|z|<1$ under the transformation $u=z /(1-z)^{2}$. Clearly there is a $\rho<1$ such that $F(z)$ has exactly $p$ zeros in $0 \leqq|z| \leqq \rho$, and no zeros in $\rho<|z|<1$.

Thus for $\rho<r<1$

$$
\frac{1}{2 \pi i} \oint_{|z|=r} \frac{F^{\prime}(z)}{F(z)} d z=p
$$

But the integral is just $i \Delta \arg F\left(r e^{i \theta}\right)$ and so $\Delta \arg F\left(r e^{i \theta}\right)=2 \pi p$. From this and the fact that $F(z)$ is starlike, it is clear that $F(z)$ is starlike of order $p$, and hence $\Im F\left(r e^{i \theta}\right)$ changes sign $2 p$ times. Therefore $F(z) \in T(p)$ and Theorem 2 is proved. From Theorem 2 and Lemma 7 we have immediately the following theorems.

THEOREM 3. If $f(z)$ given by (1.1) is p-valently starlike with respect to a point on the real axis, and if all the coefficients are real, then (1.3) is valid for 
every $n>p \geqq 1$, and this bound is sharp in all of the variables $\left|b_{1}\right|,\left|b_{2}\right|, \cdots$, $\left|b_{p}\right|$.

THEOREM 4. If $f(z)$ given by (1.1) is p-valently convex, and if all the coefficients are real then for $n>p \geqq 1$

$$
\left|b_{n}\right| \leqq \sum_{k=1}^{p} \frac{k}{n} D(p, k, n)\left|b_{k}\right|
$$

and this bound is sharp in all of the variables $\left|b_{1}\right|,\left|b_{2}\right|, \cdots,\left|b_{p}\right|$.

This settles a conjecture first advanced in [4], in the special case that all the coefficients are real.

\section{REFERENCES}

1. M. Biernacki, Sur les fonctions multivalentes d'ordre p, C. R. Acad. Sci. Paris vol. 203 (1936) pp. 449-451.

2. J. Dieudonné, Recherches sur quelques problèmes relatifs aux polynomes et aux fonctions bornées d'une variable complexe, Ann. École Norm. (3) vol. 48 (1931) pp. 247-358.

3. A. W. Goodman, On some determinants related to p-valent functions, Trans. Amer. Math. Soc. vol. 63 (1948) pp. 175-192.

4. - On the Schwarz-Christoffel transformation and p-valent functions, Trans. Amer. Math. Soc. vol. 68 (1950) pp. 204-223.

5. M. S. Robertson, A representation of all analytic functions in terms of functions with positive real parts, Ann. of Math. vol. 38 (1937) pp. 770-783.

6. - The variation of the sign of $V$ for an analytic function $U+i V$, Duke Math. $J$. vol. 5 (1939) pp. 512-519.

7. - - Star center points of multivalent functions, Duke Math. J. vol. 12 (1945) pp 669-684.

8. W. Rogosinski, Über positive harmonische Entwicklungen und typisch-reelle Potenzreihen, Math. Zeit. vol. 35 (1932) pp. 93-121.

9. O.Szász, Über Funktionen, die den Einheitskreis schlicht abbilden, Jber. Deutschen Math. Verein. vol. 42 (1932) pp. 73-75.

\section{UNIVERSITY OF KENTUCKY, LEXINGTON, KY. \\ RUTGERS UNIVERSITY, \\ New Brunswick, N.J.}

